\title{
Dependence of the Acceleration of the Outer Edge of a Stellar Envelope on Supernova Explosion Energy
}

\author{
D. K. Nadyozhin ${ }^{1,2 *}$ A. V. Karamyan ${ }^{3}$, and E. K. Grasberg ${ }^{4}$ \\ ${ }^{1}$ Institute for Theoretical and Experimental Physics, \\ ul. Bolshaya Cheremushkinskaya 25, Moscow, 117218 Russia \\ ${ }^{2}$ Max-Planck-Institut für Astrophysik, Karl-Schwarzschild-Str. 1, Postfach 1317, D-85741 Garching, Germany \\ ${ }^{3}$ Moscow Institute of Physics and Technology, Institutskii per. 9, Dolgoprudnyi, Moscow oblast, 141700 Russia \\ ${ }^{4}$ Institute of Astronomy, Latvian University, Riga, Latvia
}

Received October 9, 2008

\begin{abstract}
Based on numerical simulations of a supernova explosion, we investigate the shock-wave breakout through the stellar surface. The computations have been performed in a wide range of explosion energies and presupernova masses. The results are compared with the classical Gandelman-FrankKamenetsky self-similar solution. We have determined the dependence of an arbitrary coefficient in the selfsimilar solution on the explosion energy and presupernova structure. The derived analytical approximation formula for this coefficient can be used to estimate the supernova explosion energy from such parameters of the ejected envelope determined from astronomical observations as its maximum expansion velocity and the density distribution along its outer edge. The formula may prove to be also useful in studying the $\mathrm{X}$-ray and gamma-ray bursts that accompany the shock-wave breakout through the surface of compact presupernovae.

PACS numbers : 97.60. Bw; $95.30 . \mathrm{Lz}$

DOI: $10.1134 / \mathrm{S} 1063773709060048$
\end{abstract}

Key words: supernovae, shock waves.

\section{INTRODUCTION}

A supernova $(\mathrm{SN})$ explosion begins with the breakout of a strong shock wave (SW) through the stellar surface. Since the matter density decreases rapidly with decreasing SW distance to the stellar surface, the SW-transported energy is transferred to a progressively smaller amount of matter. Thus, the SW breakout through the stellar surface is cumulative in nature and is described by a self-similar solution of hydrodynamic equations under certain conditions (Gandelman and Frank-Kamenetsky 1956; Sakurai 1960). This solution contains an arbitrary parameter $K_{2}$ that defines the SW amplitude in the outer stellar layers.

After the SW breakout, the stellar envelope undergoes an additional acceleration in a rarefaction wave. This stage also proceeds in a self-similar regime (Sakurai 1960; Litvinova and Nadyozhin 1990), for which the initial conditions are determined by the previous SW breakout stage. Therefore, $K_{2}$ also enters into the description of this stage.

\footnotetext{
*E-mail: Nadezhin@itep.ru
}

The self-similar solution has been repeatedly applied in solving various problems related the SW breakout, as, for example, in Bisnovaty-Kogan et al. (1975), Imshennik and Nadyozhin (1988a, 1988b), and Chevalier (1992), where simple power-law approximations were used for $K_{2}$ as a function of the SN explosion energy $E_{\exp }$. However, how accurate these approximations are in a wide range of $E_{\exp }$ and how they depend on the presupernova model has remained unclear up until now.

The main goal of this paper is to elucidate the dependence of $K_{2}$ on the SN explosion energy $E_{\exp }$ and on the presupernova structure (in particular, on its mass and radius), given the variations in all these parameters over wide ranges characteristic of compact core-collapse SNe.

We numerically simulated the propagation of a SW from the stellar center to its breakout through the surface. The dependence of the post-shock matter velocity on the SW distance to the surface obtained in our calculations is compared with that predicted by the self-similar solution. This allows the numerical value of $K_{2}$ to be determined at a given explosion 
energy $E_{\exp }$. The results are presented in the form of easy-to-use (in applications) approximation formulas for the function $K_{2}\left(E_{\exp }\right)$ calculated for polytropic gaseous spheres with indices $n=1.5$ and 3 and for the evolutionary model of the presupernova 1987A computed by Woosley and Weaver (1992) and accessible on the Internet.

The SW propagation in extended SN envelopes was studied in detail by Matzner and McKee (1999). Here, we consider a more modest problem-the problem on the parameters of the self-similar solution that describes the SW propagation in the outermost layer of a star with a thickness of $10-20 \%$ of its radius. In the self-similar solution, we neglect the small corrections for the deviations from a plane geometry (Kazhdan and Murzina 1992). This is justified by the fact that applying these corrections would require much more stringent constraints on the approximation accuracy of numerical calculations: we would have to abandon the simple formula (24), with an accuracy of $\pm 5 \%$, and to take into account the weak dependence of the coefficient $\xi$ on the stellar mass.

SN 1987A exploded in the Large Magellanic Cloud had long remained the only SN whose optical emission was recorded approximately one day after the SW breakout. At present, there are several SNe discovered immediately after or even at the time of SW breakout (see, e.g., Soderberg et al. 2008; Chevalier and Fransson 2008; Quimby et al. 2007). Therefore, detailed studies of the SW breakout are of special interest.

\section{BASIC EQUATIONS \\ AND THE COMPUTATIONAL METHOD \\ Equations of Radiation Hydrodynamics}

We use the equations of spherically symmetric hydrodynamics with gravity in Lagrangian coordinates:

$$
\begin{gathered}
\frac{\partial r}{\partial t}=u \\
\frac{\partial u}{\partial t}=-r^{2} \frac{\partial(P+Q)}{\partial m}-\frac{m}{r^{2}} \\
\frac{\partial r^{3}}{\partial m}=\frac{3}{\rho} \\
\frac{\partial E}{\partial t}+(P+Q) \frac{\partial}{\partial t}\left(\frac{1}{\rho}\right)=C_{1} \frac{\partial}{\partial m}\left(\frac{r^{4}}{\kappa} \frac{\partial T^{4}}{\partial m}\right), \\
P=\rho T+B T^{4} \\
E=\frac{3}{2} T+3 B \frac{T^{4}}{\rho}
\end{gathered}
$$

where $r$ is the radius of the spherical layer that bounds mass $m ; u$ is the matter velocity; $P, \rho$, and $T$ are the pressure, density and temperature, respectively; $E$ is the specific energy; $Q$ is the artificial NeumannRichtmyer viscosity; and $\kappa$ is the opacity, which is determined by the Thomson scattering in our case, $\kappa=0.2(1+X)$. We took the hydrogen mass fraction $X$ in our calculations to be 0.7 .

Equations (1)-(6) are written in the following system of units (Nadyozhin and Frank-Kamenetsky 1964):

$$
\begin{gathered}
r \longrightarrow R, \quad m \longrightarrow M, \\
T \longrightarrow \mu \frac{m_{u}}{k} \frac{G M}{R}, \quad P \longrightarrow \frac{G M^{2}}{4 \pi R^{4}}, \\
\rho \longrightarrow \frac{M}{4 \pi R^{3}}, \quad u \longrightarrow \sqrt{\frac{G M}{R}}, \\
E \longrightarrow \frac{G M}{R}, \quad t \longrightarrow R \sqrt{\frac{R}{G M}},
\end{gathered}
$$

where $M$ is the total mass of the star, $R$ is its radius, $G$ is the gravitational constant, $m_{u}$ is the atomic mass unit, $k$ is the Boltzmann constant, and $\mu$ is the mean molecular mass. The viscosity $Q$ is measured in the same units as the pressure.

The equations of state (5) and (6) for completely ionized matter and equilibrium radiation include the dimensionless coefficient $B$ :

$$
\begin{aligned}
B & =\frac{4 \pi a}{3 G}\left(\frac{m_{u} G}{k}\right)^{4}\left(\mu^{2} M\right)^{2} \\
& =0.78096\left(\mu^{2} \frac{M}{M_{\odot}}\right)^{2},
\end{aligned}
$$

where $a$ is the radiation density constant.

The coefficient $C_{1}$ of the term that describes the radiative heat conduction in Eq. (4) has the dimensions of opacity $\kappa\left(\mathrm{cm}^{2} \mathrm{~g}^{-1}\right)$ and is defined by the expression

$$
C_{1}=\frac{1}{3} 16 \pi^{2} a c\left(\mu \frac{m_{u}}{k}\right)^{4} G^{5 / 2} R^{5 / 2} M^{1 / 2},
$$

where $c$ is the speed of light.

The independent dimensionless Lagrangian coordinate $m$ varies over the range $m_{1} \leqslant m \leqslant 1$.

\section{Boundary Conditions}

Inside the star at $m=m_{1}: r=r_{1}$ and $u=0$.

On the stellar surface at $m=1: \rho=0$ and $P=B T^{4}$.

In addition, the following condition for the temperature gradient should be used on a freely emitting stellar surface (Imshennik and Nadyozhin 1964):

$$
-C_{2} \frac{r^{2}}{\kappa} \frac{\partial T^{4}}{\partial m}=T^{4}, \quad m=1
$$


where the coefficient $C_{2}$, with the dimensions of opacity, is defined by the formula

$$
C_{2}=\frac{8 \pi}{3} \frac{R^{2}}{M}
$$

\section{Initial Conditions}

As the initial stellar structure models, we used hydrostatically equilibrium polytropic gaseous spheres with indices $n=1.5$ and 3 as well as the evolutionary model of the presupernova 1987A by Woosley and Weaver (1992), which was designated by them as I22n2a_22111 (below referred to as the WW model).

For the polytropic gaseous spheres, we must set $m_{1}=0$ and $r_{1}=0$. Thus, these models disregard the presence of a compact iron core in core-collapse SNe.

The WW model has a mass and a radius of $22.12 M_{\odot}$ and $63.0 R_{\odot}$, respectively. It contains an iron core with a mass of $1.344 M_{\odot}$ and a radius of $1.44 \times 10^{8} \mathrm{~cm}$, whose gravitational collapse will lead to the expulsion of the outer envelope during a SN explosion. For our purposes, it will suffice to associate precisely these values of $m$ and $r$ with the inner boundary of the envelope. Therefore, we take $m_{1}=6.076 \times 10^{-2}$ and $r_{1}=3.286 \times 10^{-5}$ for the WW model. In addition, for our purposes, it turned out to be necessary to make a modification in the description of the structure of the outer atmosphere for this model (see the next section).

At the initial instant $(t=0)$, the star is in hydrostatic equilibrium and, hence, the velocity $u(m)=$ 0 everywhere inside it. We simulated the explosion of the star in our computations through the release of an energy $\Delta E$ at $t=0$ due to an instantaneous temperature rise in a small mass $\delta m$ located either at the stellar center (polytropic models) or immediately adjacent to $m_{1}$ in the WW model. The mass $\delta m$ occupies 15 computational mass zones and is only $3.6 \times 10^{-7}$ for the polytropic models (an almost point explosion) and $6.2 \times 10^{-3}$ for the WW model $(10 \%$ of the iron core mass). We will call the total positive energy of the star disturbed at $t=0$ the explosion energy $E_{\text {exp }}$. Obviously,

$$
E_{\text {exp }}=\Delta E+E_{\mathrm{T}}+E_{\mathrm{g}},
$$

where $E_{\mathrm{T}}$ and $E_{\mathrm{g}}$ are the initial total thermal and gravitational energies of a hydrostatically equilibrium star for the polytropic models and a hydrostatically equilibrium envelope $\left(m_{1} \leqslant m \leqslant 1\right)$ for the WW model. When $t \rightarrow \infty$, the gravitational and thermal energies become zero and, according to the energy conservation law (which holds good in our numerical calculations), the entire energy $E_{\exp }$ transforms into the kinetic energy of the ejecta.

\section{Computational Method}

The computations were performed using a crosstype difference scheme with an explicit calculation of the new radius, velocity, and density in Eqs. (1)-(3) and an implicit calculation of the new temperature in Eq. (4) (sweep method).

When computing the SW propagation in the polytropic models, we divided the star into 1000 mass zones. The variable dimensionless mass step $\Delta m$ was chosen to be small near the center (the total mass of the first twenty zones is only $\sim 10^{-6}$ ). In the outer part of the star, $\Delta m$ decreased from $1.4 \times 10^{-3}$ for the $J=650$ zone to $\sim 10^{-12}$ near the surface $(J=1000)$. Note that these 350 outer zones contain $2.2 \%$ of the stellar mass and occupy 35 and $12 \%$ of the radius for the $n=3$ and 1.5 polytropes, respectively.

The WW model contains 432 mass zones, 109 of which are accounted for by the iron core located outside the region of our computations. Unfortunately, the division of the outer part of this model is too coarse for our purposes - the outer layer $0.77<r \leqslant 1$ is accounted for by only five mass zones. Therefore, in the range of radii $0.67<r \leqslant 1$, we supplemented the WW model by a polytropic atmosphere with a peripheral polytropic index $n=3$ by dividing it into 300 mass zones corresponding to a uniform division in radius. The zone mass gradually decreases from $\Delta m=1.2 \times 10^{-3}$ at $r=0.67$ in the WW model to $\Delta m=4.5 \times 10^{-13}$ at $r=1$. As a result, the WW model modified in this way contains 616 mass zones within our computational range $m_{1} \leqslant m \leqslant 1$.

This modification is based on the fact that the decrease in mass with depth in the atmosphere can be neglected with a high accuracy in the extended outer stellar layers. Thus, for example, only $\sim 2 \%$ of the total stellar mass is contained in the outer layer $0.65<r<$ 1 of the $n=3$ polytrope. Under such circumstances and the additional condition $P=A \rho^{1+1 / n}$, the hydrostatic equation has the well-known analytical solution

$$
\begin{gathered}
\rho=K_{1}\left(\frac{1}{r}-1\right)^{n}, \quad K_{1} \equiv[(n+1) A]^{-n}, \\
P=\frac{1}{n+1} K_{1}\left(\frac{1}{r}-1\right)^{n+1} .
\end{gathered}
$$

Approximation (13) for the WW model is shown in Fig. 1. The short vertical line corresponds to $r=$ 0.67 , while the solid line to the left of it $(r>0.67)$ corresponds to law (13) with $n=3, A=0.3092$, and $K_{1}=0.5286$ used in our calculations. For a polytropic gaseous sphere with $n=3$, we have $A=$ 0.15654 and $K_{1}=4.073$.

ASTRONOMY LETTERS Vol. 35 No. $6 \quad 2009$ 


\section{SELF-SIMILAR SOLUTION}

Constancy of the adiabatic index $\gamma$ behind the front of a strong SW (in our case of a radiation-dominated $\mathrm{SW}, \gamma=4 / 3$ ) and a power-law dependence of the density on the SW distance to the stellar surface, which follows from (13) at $r$ close to unity,

$$
\rho=K_{1} x^{n}, \quad x \equiv 1-r
$$

are required for the existence of a self-similar solution.

According to the self-similar solution, the SW front velocity $D$ increases indefinitely with decreasing SW distance $x_{\mathrm{sw}}$ to the stellar surface:

$$
D=\frac{d r_{\mathrm{sW}}}{d t}=-\frac{d x_{\mathrm{sW}}}{d t}=K_{2} x_{\mathrm{sW}}^{-\lambda}, \quad K_{2}>0,
$$

where $\lambda>0$ depends only on $n$ and $\gamma$ and is defined as an eigenvalue when finding the self-similar solution and $K_{2}$ is an arbitrary constant.

Grasberg (1981) provided a wide spectrum of $\lambda$ values calculated for various $n$ and $\gamma$. Here, we will need $\lambda$ for $\gamma=4 / 3$ and $n=3$ and 1.5 , which are 0.5572 and 0.2863 , respectively.

The matter velocity $u$ at the front of a strong SW is related to $D$ by

$$
u=\frac{2}{\gamma+1} D=\frac{2}{\gamma+1} K_{2} x_{\mathrm{sw}}^{-\lambda} .
$$

At the time of SW breakout through the stellar surface $\left(x_{\mathrm{sw}}=0\right)$, the velocity at the $\mathrm{SW}$ front becomes infinite, while all physical quantities in the inner layers of the envelope are power functions of the distance to the stellar surface at this time, which we will denote by $x_{0}$ :

$$
\begin{gathered}
\rho\left(x_{0}\right)=K_{1} x_{0}^{n} h_{\infty}, \\
P\left(x_{0}\right)=K_{1} K_{2}^{2} x_{0}^{n-2 \lambda} g_{\infty}, \\
u\left(x_{0}\right)=-\frac{\mathrm{d} x}{\mathrm{~d} t}=K_{2} x_{0}^{-\lambda} f_{\infty},
\end{gathered}
$$

where the coefficients $h_{\infty}, g_{\infty}$, and $f_{\infty}$ were tabulated by Grasberg (1981) for various $n$ and $\gamma$.

After the SW breakout, the matter expands into a vacuum and accelerates in a rarefaction wave until the phase of a free inertial expansion begins, when the velocity $u$ of each mass layer initially located at distance $x_{0}$ from the surface at the time of SW breakout no longer changes with time and its radius $r$ is proportional to the time: $r=u t$. The initial phase of expansion into a vacuum is described by a different self-similar solution (Sakurai 1960; Litvinova and Nadyozhin 1990). According to the latter, the velocity of each mass layer increases by the same factor by the time of transition to the state of a free expansion:

$$
u=\beta(n, \gamma) u\left(x_{0}\right)
$$

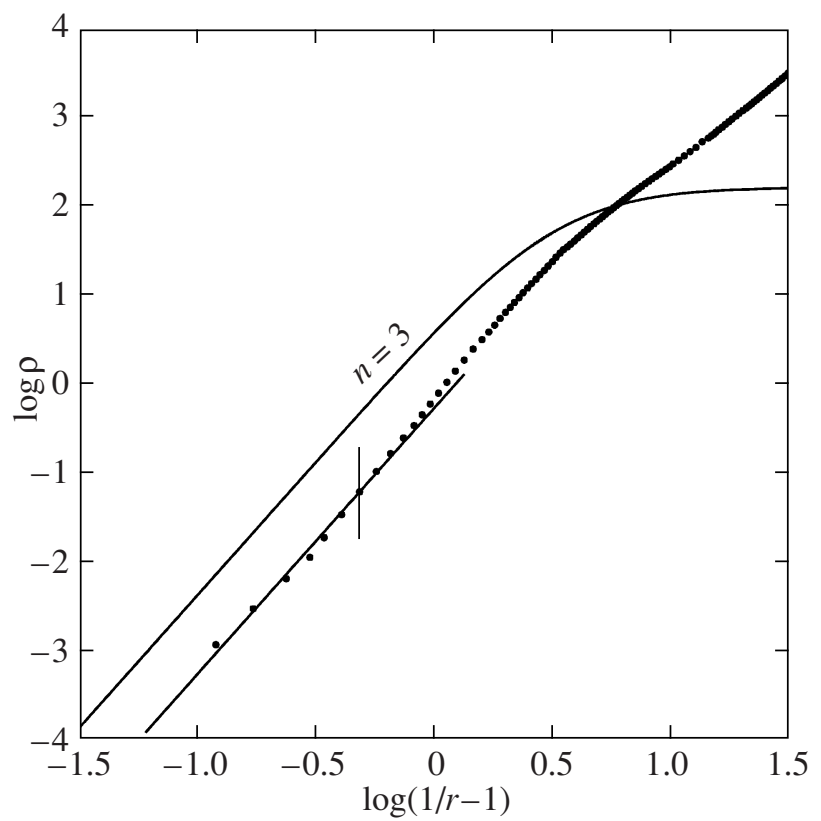

Fig. 1. Approximation of the density distribution in the outer part of the envelope in the WW evolutionary model (filled circles) by a polytropic law with an index $n=3$ (to the left of the short vertical line). The density distribution in a polytropic gaseous sphere with $n=3$ is shown for comparison.

Given the mass conservation law and the relation $r=$ $u t$, an expression for the density distribution in a freely expanding envelope can be derived from Eqs. (18), (20), and (21):

$$
\begin{gathered}
\rho=\frac{\mathcal{B}}{r^{3} u^{s}}=\frac{\mathcal{B} t^{s}}{r^{3+s}}=\frac{\mathcal{B}}{t^{3} u^{3+s}}, \\
r=u t, \quad s \equiv \frac{n+1}{\lambda} \\
\mathcal{B}=\frac{1}{\lambda} h_{\infty} K_{1}\left(\beta f_{\infty} K_{2}\right)^{s} .
\end{gathered}
$$

Equations (22) and (23) are written in our dimensionless units. Their detailed derivation can be found in Nadyozhin (1994). Unfortunately, the factor $4 \pi$, which was replaced by unity in Eq. (23), is present by mistake in the final expression for $\mathcal{B}$ (Eq. (1.25)) from the above paper. Since $K_{1}$ and $K_{2}$ have the dimensions of density and velocity, respectively (see Eqs. (15) and (16)), the right-hand side of (23) can be multiplied by $R^{3}$ ( $R$ is the presupernova radius) and Eqs. (22) and (23) will then be valid for any system of units if, of course, $K_{1}$ and $K_{2}$ are measured in the same units as $\rho$ and $u$, respectively.

\section{NUMERICAL CALCULATIONS}

For both polytropic models $(n=1.5,3)$, we performed computations at 13 explosion energies $E_{\exp }$, 


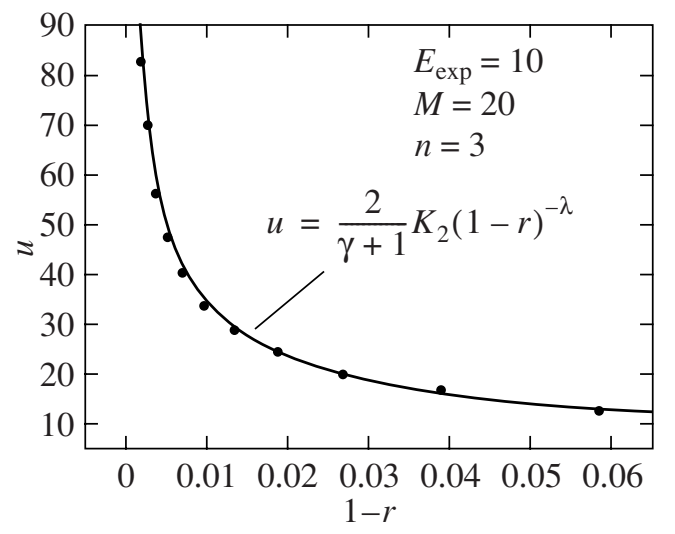

Fig. 2. Matter velocity at the SW front versus distance to the stellar surface located to the left for a polytropic model $(n=3)$ with a mass of $2-M_{\odot}$ and a dimensionless explosion energy $E_{\text {exp }}=10$.

in the range from 1 to 100 in dimensionless units $\left(G M^{2} / R\right.$ serves as the natural unit of energy). The dimensionless gravitational energies of the initial hydrostatically equilibrium polytropic models are -1.5 and $-6 / 7$ for $n=3$ and 1.5 , respectively. At each $E_{\text {exp }}$, we also performed a series of computations for eight stellar masses $M$ in the range from $10 M_{\odot}$ to $50 M_{\odot}$.

These computations revealed that the effect of radiative heat conduction on the SW propagation for compact presupernovae (with radii $R \lesssim 100 R_{\odot}$ ) is noticeable only in the outermost layers of the envelope with a thickness of several percent of the stellar radius, where the SW directly breaks out through the stellar surface (see the next section). Therefore, for our purpose (determining $K_{2}$ ), we can restrict ourselves to the adiabatic approximation by setting $C_{1}=0$ in the energy equation (4). Most of the computations for the polytropic models were performed precisely in this approximation.

For the WW evolutionary model, we performed computations at six values of $E_{\exp }=6.64,14.8,23.9$, $43.1,77.5,128$ (all with radiative heat conduction). Since $G M^{2} / R=2.95 \times 10^{49}$ erg for the WW model, this corresponds to a change in explosion energy in the range $(0.2-3.8) \times 10^{51} \mathrm{erg}$.

It should be noted that the system of equations (1)-(6) in the adiabatic approximation not only does not contain the constant $C_{1}$ but also does not need the boundary condition (10). Only one arbitrary parameter remains, $\mu^{2} M$, which determines the constant $B$ in the equation of state. If the initial conditions written in the system of units (7) contain no arbitrary parameters (except the explosion energy $E_{\exp }$ ), then the solution of the problem in dimensionless units depends only on $\mu^{2} M$ and $E_{\exp }$ and does not depend on the stellar radius $R$. This case applies to the polytropic models. Therefore, the dimensionless values of $K_{2}$ for the $n=1.5$ and 3 polytropic models obtained in our computations do not depend on $R$ and are weakly sensitive to variations in the stellar mass, which enters into the problem via the parameter $\mu^{2} M$. This result tested in our numerical calculations can be explained by the fact that the equilibrium radiation pressure exceeds considerably the matter pressure in the entire shocked region. If the pressure and specific energy of the matter are neglected altogether, then the two equations of state (5) and (6) merge into one, $E=3 P / \rho$, and the parameter $\mu^{2} M$ drops out of the system.

Figure 2 shows an example of the calculation (without radiative heat conduction) of $K_{2}$ for the $n=$ 3 polytropic model at an explosion energy $E_{\exp }=$ 10 and a mass of $20 M_{\odot}$. The points indicate the velocity at the SW front obtained in our hydrodynamic computation at consecutive instants of time as the SW approached the stellar surface. The solid curve represents dependence (17) at $\gamma=4 / 3$ and $\lambda=0.5572$ from the self-similar solution. The value of $K_{2}$ is chosen from the condition that the curve corresponds most closely to the calculated velocities. Such a procedure of determining $K_{2}$ was used for all our computations: all values of $E_{\exp }$ and $M$ and, when the radiative heat conduction is taken into account, $R$ and for all of the models considered here. It turned out that the dependences $K_{2}\left(E_{\exp }\right)$ found in this way for all three models could be approximated by the same law

$$
K_{2}=\xi \sqrt{E_{\exp }},
$$

where the coefficient $\xi$ depends on the model,

$$
\begin{gathered}
\xi(n=1.5)=0.722, \\
\xi(n=3)=0.921, \quad \xi(W W)=1.62 .
\end{gathered}
$$

The uncertainty in the coefficients $\xi$ for the $n=$ 1.5 and 3 polytropes is $\pm 5 \%$. It arises mainly from the weak dependence of the calculated $K_{2}$ on the stellar mass $M$ and is the price to pay for neglecting this dependence when the computational data are approximated. The approximation formula (24) for the WW model has an accuracy of at least $5 \%$ for dimensionless explosion energies in the range $10 \lesssim$ $E_{\exp } \lesssim 130$. At $E_{\exp }<10$, the accuracy of approximation (24) deteriorates. Thus, for example, the calculated value of $K_{2}$ for $E_{\exp }=6.64$ exceeds the value that follows from (24) by $20 \%$. This small deviation from dependence (24) for the WW model probably results from an additional SW acceleration due to the energy transfer by radiative heat conduction into the outer stellar layers. This effect does not manifest itself in the polytropic models, since the density in their 


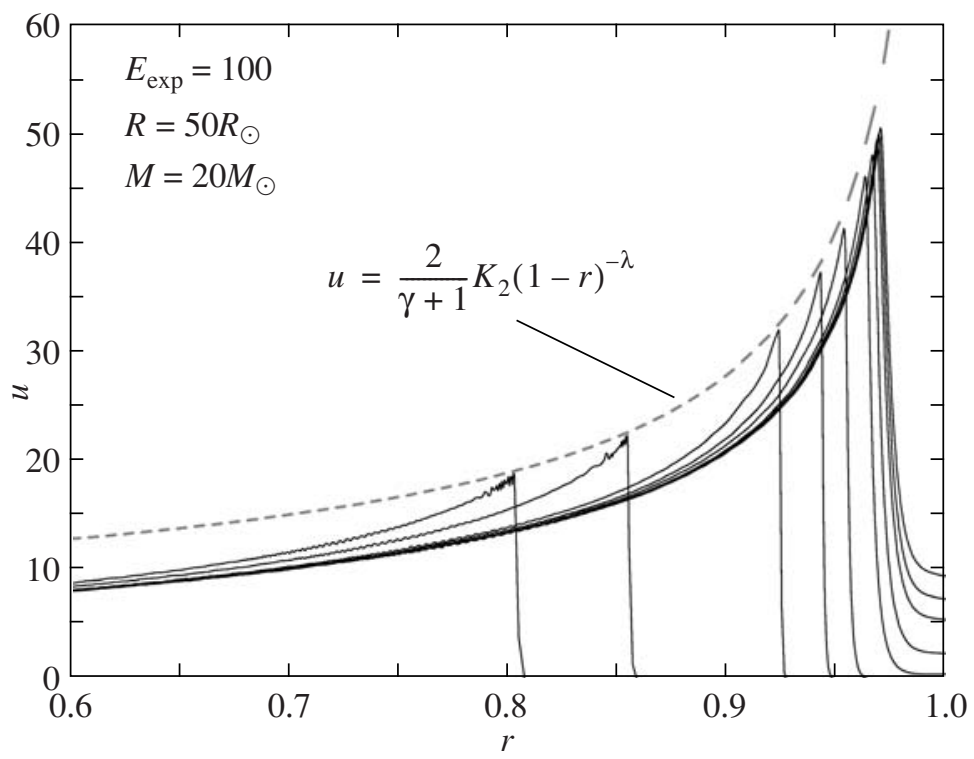

Fig. 3. Dimensionless matter velocity versus radius at various instants of time for a polytrope $(n=3)$ with a mass of $20 M_{\odot}$, an initial radius of $50 R_{\odot}$, and explosion energy $E_{\exp }=100\left(3.04 \times 10^{51} \mathrm{erg}\right)$.

envelopes (at $0.5 \lesssim r<1$ ) is much higher than that in the WW model (Fig. 1).

After the passage to dimensional quantities, Eq. (24) takes the form

$$
K_{2}=\xi_{0} \sqrt{\frac{E_{\exp }}{M}} .
$$

If $E_{\exp }$ and $M$ are measured in units of $10^{51} \mathrm{erg}$ and $M_{\odot}$, respectively, then the coefficient $\xi_{0}$ in units of $\mathrm{cm} \mathrm{s}^{-1}$ is

$$
\xi_{0}(n=1.5)=5.1 \times 10^{8},
$$

$$
\xi_{0}(n=3)=6.5 \times 10^{8}, \quad \xi_{0}(\mathrm{WW})=1.15 \times 10^{9} .
$$

Note that Eq. (26) for the WW model virtually coincides with the $K_{2}$ estimate that was used by Imshennik and Nadyozhin (1988a) to interpret the observations of SN 1987A.

\section{MAXIMUM VELOCITY}

The self-similar solution is inapplicable in the outermost stellar layers, where the density is so low that the characteristic time of energy outflow from the SW due to radiative diffusion is shorter than the time left before the SW breakout through the stellar surface. This effect restricts the increase in the velocity at the SW front, which in the self-similar solution formally tends to infinity as the distance between the SW front and the stellar surface decreases. As a result, the final velocity of the outer edge of the expanding envelope turns out to be limited from above.
The optical width of the front of a radiationdominated SW is known (see Imshennik and $\mathrm{Na}$ dyozhin (1988a, 1988b) and references therein) to be defined by the relation

$$
\delta \tau_{\mathrm{sw}} \approx \frac{c}{D} .
$$

The optical distance from the SW to the surface is defined by the integral

$$
\tau_{\mathrm{sw}}=\int_{r_{\mathrm{sw}}}^{R} \kappa \rho \mathrm{d} r,
$$

where $r_{\mathrm{sw}}$ is the radius of the SW front. When $\tau_{\mathrm{sw}}$ approaches $\delta \tau_{\text {sw }}$, an outward flux "breakout" occurs and the self-similar solution becomes inapplicable. Thus, the applicability condition for the self-similar solution can be written as

$$
\tau_{\mathrm{sw}} \gtrsim \delta \tau_{\mathrm{sw}} .
$$

Using Eqs. (15), (16), (17), and (26), we can write inequality (30) as

$$
x_{\mathrm{sw}} \gtrsim x_{\mathrm{min}},
$$

where $x_{\mathrm{sw}} \equiv 1-r_{\mathrm{sw}} / R$ is the dimensionless distance from the SW to the stellar surface. For $x_{\min }$, we have

$$
\begin{gathered}
x_{\min }^{n+1-\lambda}=1.53 \times 10^{-10} \\
\times \frac{(n+1)[(n+1) A]^{n}}{1+X} \frac{c}{\xi_{0}} \frac{R^{2}}{\sqrt{M E_{\exp }}},
\end{gathered}
$$

where $M$ and $R$ are given in solar units and $E_{\exp }$ is given in $10^{51} \mathrm{erg}$. 


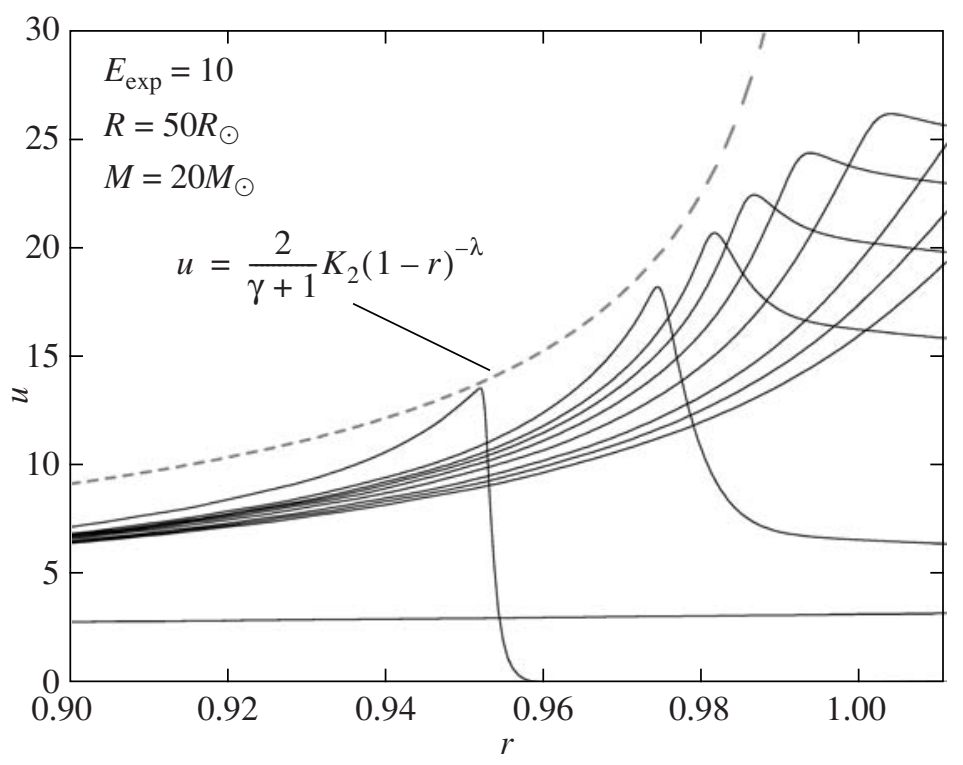

Fig. 4. Same as Fig. 3 for the explosion energy $E_{\exp }=10\left(3.04 \times 10^{50} \mathrm{erg}\right)$.

Figures 3 and 4 show the velocity distributions in radius in the outer $\mathrm{SN}$ layers for the $n=3$ polytropic model that were computed by taking into account the radiative heat conduction for the dimensionless explosion energies $E_{\exp }=100$ and 10, respectively.

The dashed curve represents the locus of points that correspond to the velocities at the SW front (Eq. (17)). The parameter $K_{2}$ was calculated from Eq. (24) for the corresponding dimensionless explosion energy $E_{\text {exp }}$.

We see that the self-similar solution describes the SW propagation with an adequate accuracy up to the distance to the stellar surface $x_{\min }$ defined by Eq. (32), 0.020 and 0.027 for Figs. 3 and 4, respectively.

In Fig. 4, we clearly see the SW transformation in the outer layer of thickness $x_{\min }$ into a rarefaction wave.

We can now estimate the maximum matter velocity $u_{\max }$ at the SW front immediately before the SW transformation into a rarefaction wave, i.e., at $x_{\mathrm{sw}}=$ $x_{\min }$. Substituting $x_{\min }$ from (32) into (17) yields

$$
\begin{aligned}
& u_{\max }=\frac{2}{\gamma+1} K_{2} x_{\min }^{-\lambda} \\
= & \frac{2}{\gamma+1} \xi_{0}\left(M / M_{\odot}\right)^{-0.5} \\
\times & \left(E_{\exp } / 10^{51} \mathrm{erg}\right)^{0.5} x_{\min }^{-\lambda} .
\end{aligned}
$$

It follows from (32) and (33) that $u_{\max }$ is a power function of $E_{\text {exp }}, M$, and $R$ :

$$
\begin{aligned}
u_{\max } & \propto E_{\exp }^{0.5 \frac{n+1}{n+1-\lambda}} M^{-0.5 \frac{n+1-2 \lambda}{n+1-\lambda}} R^{\frac{-2 \lambda}{n+1-\lambda}} \\
& =E_{\exp }^{0.581} M^{-0.419} R^{-0.324}, \quad n=3 .
\end{aligned}
$$

The dimensionless mass $\delta m$ of the outer layer $0<$ $x<x_{\min }$ accelerated to $u_{\max }$ can be determined from the relation

$$
\delta m \approx \frac{K_{1}}{n+1} x_{\min }^{n+1} .
$$

For the polytropic model $(n=3)$ shown in Fig. 4 , the dimensionless value of $u_{\max }=18.6$ defined by Eqs. (17), (24), and (25) is in good agreement with this figure. In dimensional units, $u_{\max }=5100 \mathrm{~km} \mathrm{~s}^{-1}$.

For the WW model at an explosion energy of $1 \times 10^{51}$ erg, we obtain $x_{\min }=0.0397$ and $u_{\max }=$ $12700 \mathrm{~km} \mathrm{~s}^{-1}$. After the additional acceleration in the rarefaction wave, the velocity increases to $\beta u_{\max }$ (Eq. (21)). At $\beta \approx 1.6$ (Litvinova and Nadyozhin 1990 ), the maximum velocity of the outer edge of the SN 1987 A envelope can reach $\approx 20000 \mathrm{~km} \mathrm{~s}^{-1}$. It follows from $(35)$ that $\delta m \approx 3.3 \times 10^{-7}$ and, hence, the mass of the matter with a velocity close to $u_{\max }$ is $M \delta m \approx 7.3 \times 10^{-6} M_{\odot}$.

The most detailed hydrodynamic model for the SN 1987A explosion was developed by Utrobin (1993, 2004). Immediately before its explosion, the star had a mass of $19.58 M_{\odot}$ and a radius of $46.8 M_{\odot}$. In the outer envelope of this model, the hydrogen mass fraction is $X=0.55$ and the density distribution can be approximated by a polytropic law $(n=3)$ with a dimensionless parameter $A \approx 0.49$ in Eq. (13). The values of $\xi \approx 2.12$ and $\xi_{0} \approx 1.5 \times$ $10^{9} \mathrm{~cm} \mathrm{~s}^{-1}$ in Eqs. (24) and (26) correspond to the hydrodynamic computation shown in Fig. 12 from Utrobin (2004) and designated there as N1o. Using these data, we obtain $x_{\min } \approx 0.048, u_{\max } \approx$ 
$16000 \mathrm{~km} \mathrm{~s}^{-1}, \beta u_{\max } \approx 25000 \mathrm{~km} \mathrm{~s}^{-1}$, and $M \delta m \approx$ $3.5 \times 10^{-6} M_{\odot}$ for Utrobin's model, which agrees with the hydrodynamic picture presented in the mentioned Fig. 12 to within $\approx 10 \%$.

\section{CONCLUSIONS}

Our main result is the elucidation of the dependence of the parameter $K_{2}$ in the self-similar solution on the explosion energy $E_{\exp }$ and presupernova structure. We showed that when using dimensionless variables, $K_{2}$ is proportional, with an adequate accuracy, to the square root of $E_{\exp }$ (Eq. (24)), with the proportionality factor $\xi$ being independent of the presupernova radius $R$ and virtually independent of its mass $M$. In dimensional form, $K_{2}$ is proportional to the mean velocity of the expanding envelope (Eq. (26)).

Our results apply to compact $\mathrm{SNe}$ with $R \lesssim$ $100 R_{\odot}$. Equations (24) and (26) for $K_{2}$ are applicable when the explosion energy exceeds considerably the gravitational binding energy of the $\mathrm{SN}$ envelope, $E_{\exp } \gtrsim 10 G M^{2} / R$. Otherwise, the calculated values of $K_{2}$ turn out to be slightly higher than those obtained from Eqs. (24) and (26).

Our results make it possible to estimate in principle the SN explosion energy from the maximum velocity of the outer edge of the envelope (Eq. (34)) and the density $\rho$ in the regime of a free expansion (Eq. (22)) determined from astronomical observations. The latter possibility is particularly favorable due to the strong dependence of $\rho$ on $E_{\exp }, \rho \propto E_{\exp }^{\alpha}$, where $\alpha=3.59$ and 4.37 for $n=3$ and 1.5 , respectively.

\section{ACKNOWLEDGMENTS}

We wish to thank V.P. Utrobin for interest in this work and for providing additional information about his hydrodynamic model for SN 1987A of interest to us. This work was finished at the Max-PlanckInstitut für Astrophysik (Garching, Germany), which D.K. Nadyozhin thanks for hospitality and financial support.

\section{REFERENCES}

1. G. S. Bisnovaty-Kogan, V. S. Imshennik, D. K. Nadyozhin, and V. M. Chechetkin, Astrophys. Space. Sci. 35, 23 (1975).

2. R. A. Chevalier, Astrophys. J. 394, 599 (1992).

3. R. A. Chevalier and C. Fransson, arXiv:0806.0371v1 (2008).

4. G. M. Gandel'man and D. A. Frank-Kamenetsky, Dokl. Akad. Nauk SSSR 107, 811 (1956).

5. E. K. Grasberg, Astron. Zh. 58, 155 (1981) [Sov. Astron. 25, 85 (1981)].

6. V. S. Imshennik and D. K. Nadyozhin, Astron. Zh. 41, 829 (1964) [Sov. Astron. 8, 664 (1964)].

7. V. S. Imshennik and D. K. Nadyozhin, Usp. Fiz. Nauk 156, 561 (1988a).

8. V. S. Imshennik and D. K. Nadyozhin, Pis'ma Astron. Zh. 14, 1059 (1988b) [Sov. Astron. Lett. 14, 449 (1988)].

9. Ya. M. Kazhdan and M. V. Murzina, Pis'ma Astron. Zh. 18, 851 (1992)[Sov. Astron. Lett. 18, 345 (1992)].

10. I. Yu. Litvinova and D. K. Nadyozhin, Pis'ma Astron. Zh. 16, 61 (1990) [Sov. Astron. Lett. 16, 29 (1990)].

11. C. D. Matzner and C. F. McKee, Astrophys. J. 510, 379 (1999).

12. D. K. Nadyozhin, Supernovae, Les Houches, Session LIV 1990, Ed. by S. Bludman, R. Mochkovitch, and J. Zinn-Justin (Elsevier Sci., 1994), p. 571.

13. D. K. Nadyozhin and D. A. Frank-Kamenetsky, Vopr. Kosmogon. 10, 154 (1964).

14. R. M. Quimby, J. C. Wheeler, P. Höflich, et al., Astrophys. J. 666, 1093 (2007).

15. A. Sakurai, Commun. Pure Appl. Math. 13, 553 (1960).

16. A. M. Soderberg, E. Berger, K. L. Page, et al., Nature 453, 469 (2008).

17. V. P. Utrobin, Astron. Astrophys. 270, 249 (1993).

18. V. P. Utrobin, Pis'ma Astron. Zh. 30, 334 (2004) [Astron. Lett. 30, 293 (2004)].

19. S. E. Woosley and T. A. Weaver, http://www.superci.org/stellar/(1992).

Translated by V. Astakhov 